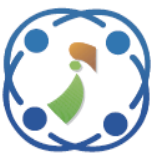

\title{
Ant Colony and Tolerable Delay Based Modified Virtual Backoff Algorithm for Wireless Sensor Networks
}

\author{
Palamakula Ramesh Babu $^{1^{*}} \quad$ Parimala Venkata Krishna $^{2}$ \\ ${ }^{1}$ Jawaharlal Nehru Technological University, Anantapur, Andhra Pradesh, India \\ ${ }^{2}$ Sri Padmavathi Mahila Viswavidyalayam, Tirupathi, Andhra Pradesh, India \\ * Corresponding author's Email: prameshjntuphd@gmail.com
}

\begin{abstract}
The MAC layer plays a major role in the enhancement of the performance of any network. Hence this paper proposes a modified virtual backoff algorithm which queues the packets based on the delay that application can tolerate and ant colony optimization technique is used to choose the best of all available forwarding nodes. Two counter referred as $\mathrm{N}_{\text {access }}$ and $\mathrm{N}_{\text {attempt }}$ are maintained for each node which is used to calculate the success rate of each node based on which the node is given the channel access of all contending nodes. Sleep scheduling is incorporated in order to enhance the energy conservation and hence increase the lifetime of the network. The performance of the proposed algorithm is proved to be better when compared to the legacy systems like VBA, S-MAC and EACAODV. The parameters used for testing are packet delivery ratio, average energy consumption, delay and number of collisions.
\end{abstract}

Keywords: Ant colony optimization, VBA, WSN, Channel access, Collision.

\section{Introduction}

The computing devices which depend on the battery power and can perform sensing operations, referred as sensors and are used as nodes in the Wireless Sensor Networks (WSN). The sensor nodes of WSN will join together for a typical application, for example, observing environmental conditions. The nodes in WSN are anticipated to be inactive state when it is not detecting any object and become active abruptly when any object is detected and are organized in an ad hoc manner. There are many features which differentiate the MAC layer of WSN from the wireless MAC, IEEE 802.11, the essential objectives like self-design and energy management and the less critical objectives like fairness of each node and delay [1].

The processing of data within the network is a vital characteristic of WSN. This characteristic of WSN is very useful in decreasing the usage of energy instead of sending the raw data completely till the end node [2-4]. To process the data within the network, accumulate and accelerating of messages need to be supported.
Advances in the hardware made to accommodate the memory, transceiver and processor in one chip and hence reduces the cost of nodes in WSN. The battery power of the sensor nodes will be less when compared to the other mobile devices which make the range of communication to be less in the case of sensor nodes. Therefore, if larger area needs to be covered then the sensor nodes required are more.

The lifetime of the network can be enhanced when the energy consumption is reduced and this plays a major role in WSN as the replacement of battery or recharging the power units is not an easy task in WSN contrasting to other wireless networks. One way of reducing the energy consumption is by reducing the communication among sensor nodes because the sensor node requires more energy to communicate when compared to process the data.

In our daily life it can be observed that the role of sensors is increasing day by day. They are being used in most of our regular activities. They became very important and needful. The components in a sensor node are sensing unit which is used to detect any object within its range of signal (e.g. temperature, heat, smoke, acoustic) or (chemical sensors), analog to digital converter which is used to convert the data 
sensed by the sensing unit which is in the form of analog to digital which facilitates in transmitting to the destination node, battery source which provides power to the node and a microcontroller (with an efficient operating system which helps in conserving energy) [7].

The significant design goal of WSN is to make the energy to be used effectively and efficiently. There are various ways proposed by the researchers to make the efficient usage of energy like optimization algorithms based on ant behaviour, particle swarm optimization and genetic algorithms [8-9].

The battery which is used in the sensor nodes cannot be recharged in most of the cases. Hence they cannot be reused and need to be disposed [10]. The case where it is possible to recharge the battery it is highly challenging as the place where the sensors normally are deployed will be secluded and hostile. Hence the energy conservation is very important in WSN which helps in increasing the lifetime of the network.

Of all the various reasons of power consumption, the data transmission and reception operation is consuming more power. There are various reasons for which energy is more consumed than the required energy. It can be said as the energy is wasted for the following reasons: Some of them are eavesdropping, listening during idle state, collision and discharging faster. During eavesdropping, the node receives the packets which are not intended to it. The probable data traffic is received when the node performs idle listening. The packets are to be transmitted again when the collision occurs and finally the discharge will be more when the packets are transmitted when the node to receive the data is not prepared.

The sensor nodes which are formed as a network are used to interpret some data about the environment and perform the specified activity (automatic sensor doors) or send the interpreted data to the server systems where it can be used to know the situation in the location where the sensor nodes are deployed (ex: border areas) [11]

One of the ways to conserve energy in WSN is by introducing duty cycle. The duty cycle makes the node to be in the sleep mode in which the nodes do not communicate for some duration and to be in active state where the node can sense and get some data. In this regard, there is a need of synchronization among neighbouring nodes. Some of the protocols that induced synchronization into MAC protocols are S-MAC, T-MAC, DSMAC, and TEEM [12-16].

There are two classifications in the MAC protocols - based on contention and based on scheduling. The protocols which are based on the contention allow all the nodes to contend for and acquire the channel access where as in the protocols which are based on the scheduling provides a schedule according to which the nodes can acquire an access to the channel [17].

The protocol is organized into various layers. The energy consumption can be optimized at any layer of the protocol stack [18-19]. The vital role of the MAC layer in the network is to eliminate the conflict among the nodes during the channel access and to identify the node to obtain the access to the channel. Furthermore, constraints like effective utilization of bandwidth and minimum delay impose added challenges to the MAC protocol design [20].

This paper proposes a modified virtual backoff algorithm which queues the packets based on the delay that application can tolerate and ant colony optimization technique is used to choose the best of all available forwarding nodes. Two counter are maintained for each node which is used to calculate the success rate of each node based on which the node is given the channel access of all contending nodes. Sleep scheduling is incorporated in order to enhance the energy conservation and hence increase the lifetime of the network.

The rest of this paper is organized as follows: section 2 gives brief description of work carried out in this field, the proposed algorithm, ACTD-M-VBA is discussed in section 3, results and discussions are carried out in section 4 and section 5 concluded the paper.

\section{Related Work}

The structural design referred as Piconet is given in [5] and this is applicable for low-power ad hoc wireless networks. The energy is conserved in Piconet by pushing the nodes into sleep mode periodically. The nodes coordinate with each other by broadcasting the node's corresponding address when it awakes from the sleep mode and becomes active to sense the objects. If a node 1 receives the message from any other node 2 , then only the communication can take place otherwise the node 1 assumes that the node 2 is in inactive state. But, this approach increases the overall completion time of the network

An adaptive rate control mechanism is proposed by Woo and Culler in [6]. They studied Carrier Sense Multiple Access (CSMA) for various configurations. The authors attempted to accomplish allocation of bandwidth in fair manner to all the nodes in the multihop network. The various MAC schemes are investigated and quantified using the motes and TinyOS platform. In this approach, the packet 
delivery ratio will be high due to the bandwidth distribution.

S-MAC [18] is a Sensor-MAC protocol which is a contention based protocol introduced a sleep mode for the nodes where the nodes will go to inactive state and will not be able to participate is sensing or forwarding in order to conserve energy. The duty cycle is static in the case of S-MAC protocol which is based on contention. The energy conservation procedure of S-MAC is in analogous to IEEE 802.11. The communication schedule in S-MAC is dynamic in nature and hence an additional control packet is required to synchronize the nodes in the network. The nodes are active in the listen period where RTS/CTS packets communication takes place. The nodes which do not receive the RTS packet in the listen period and have no data to transmit can go to the sleep period.

As the nodes in the WSN will not have any knowledge regarding the time when data will be transmitted, they keep listening to the channel all the time. Listening to the channel when there is no data transmission is referred as idle listening. Idle listening leads to wastage of energy consumption. Hence, if idle listening is minimized then energy can be conserved. This can be done by scheduling the nodes using TDMA method [21-23]. In addition to this, energy can be conserved more by making the nodes to be in active or inactive states periodically. This method is considered to be more efficient optimization method. The nodes are allotted a fixed time slot in which the node can transmit the packets or receive any data. So, the node will be in active state during its time slot. The nodes go into inactive/sleep state if the time slot is not corresponding to it. The TDMA based scheduling helps in reducing the number of collisions and hence reduces the retransmissions. The switching of the node between the active and inactive states is a trade-off as if the duration of the state is less; then the time taken to switch between the states will be more. Otherwise, if the duration of the state is more, then there is a chance of decreasing the network performance even though the time taken for switching is reduced.

The data fusion method is proposed by Donggook Kim in [24] which helps in enhancing the performance and maximizing the power conservation. Another approach is proposed Sun Dayang in [25] which aims at increasing the life time of the network. The load is reduced using the cross layer design in WSN by Chen Wei in [26].

Jing-hui Zhong tried to increase the lifespan of the network by conserving the energy using an innovative method named as Local Wakeup Scheduling (LWS) in [27]. An ant colony optimization technique based mc-ACO is utilized in order to achieve the goal. According to LWS, the nodes in the network are divided into two groups. First group will be in active state and the second group will be in sleep mode. When the nodes in the active group are becoming energy less nodes, then the modes are switched among the groups by making the nodes in the first group to be sleep mode and making the nodes in the second group to be active. This approach will create communication overhead over the network.

SSMTT, Sleep Scheduling algorithm for Multiple Target Tracking sensor networks is proposed by Bo Jiang et al in [28]. The schedule time is dynamic in nature for every node. i.e, the sleep pattern is altered based on the network situation at the time the node wakes up from the sleep mode. Similarly, whenever the node is going to the sleep mode, the wake up timer is set. When compared to the single target tracking systems, SSMTT is potential enough to conserve the energy. But, the SSMTT will increase the end to end delay at the time of packet delivery.

Medium Reservation Preamble based MAC (MRPM) is proposed in [29]. The authors attempted and succeeded in reducing the energy consumption and latency in WSN. The nodes contend for the channel during the contention period only. When the node wants to transmit the data, it wakes up during the contention period otherwise it ignores the contention period. Hence the authors made the duty cycle to be adaptive. The listen period is made short by separating the contention from it and combining SYNC packet and RTS packet. The advanced adaptive listening (AAL) procedure of MRPM is used to transmit the data multiple hops away in a single duty cycle lead to attain low latency. The proposed approach causes premature death of nodes in the network.

\section{Proposed Algorithm - Ant colony and Tolerable Delay based Modified VBA (ACTD-M-VBA)}

\subsection{Mathematical Model for M-VBA}

Let, the probability of the node ' $\mathrm{i}$ ' attempting for the access of the channel, $\mathrm{P}(\mathrm{A})$. If ' $\mathrm{m}$ ' nodes are contending for the channel simultaneously, then the probability of the node 'i' acquiring a channel, $\mathrm{P}(\mathrm{B})$. Therefore, the probability of the node attempting and acquiring the access to the channel, is $\mathrm{P}(\mathrm{A}) \mathrm{P}(\mathrm{B} \mid \mathrm{A})$. If the number of attempts is restricted to ' $\mathrm{K}$ ' then the probability of the node attempting and acquiring the access to the channel is 


$$
P(X)=P(A)^{K} P(B \mid A)^{R}, \text { where } R \leq K
$$

The above equation tells that the ' $R$ ' attempts out of ' $\mathrm{K}$ ' attempts are successful in acquiring a channel.

If the number of accesses is restricted to ' $K$ ', then the probability of the node attempting and acquiring the access to the channel is

$$
P(Y)=P(A)^{S} P(B \mid A)^{K}, \text { where } S \geq K
$$

The above equation tells that the ' $\mathrm{K}$ ' attempts out of ' $\mathrm{S}$ ' attempts are successful in acquiring a channel. When, both the Eq. 1 and Eq. 2 are compared, then it could be observed that $P(Y)>=P(X) . P(Y)=P(X)$ is possible when $\mathrm{K}=\mathrm{R}=\mathrm{S}$. Hence, starvation problem could be avoided and the restriction helps in reducing the collisions.

Initially, the probability of all nodes to acquire a channel is equal. If there are ' $m$ ' nodes among ' $n$ ' nodes are contending for the channel and ' $h$ ' nodes among ' $\mathrm{m}$ ' nodes are contending for the first time, then the probabilities of ' $\mathrm{m}$ ' nodes is given as follows:

$P_{i}(t)=\frac{\left(1-\sum_{j=1}^{m-h} P_{j(o l d)}(t)\right)}{m}$

Where $i$ is the node which is contending for the first time and $\mathrm{j}$ is the node which already contended for the channel.

$$
P_{j(\text { new })}(t)=P_{j(\text { old })}(t)+\frac{\left(1-\sum_{j=1}^{m-h} P_{j(\text { old })}(t)\right)}{m}
$$

When a node acquires a channel, then probability of the corresponding node is decreased and probability of the remaining contending nodes will be increased. The increment and decrement of probability is as shown in Eq. 3 and Eq. 4.

Assume that ' $\mathrm{m}$ ' nodes are contending for the channel at the same time and node ' $\mathrm{i}$ ' acquired a channel. Then,

$$
\begin{aligned}
& P_{i}(t+1)=(1-a) P_{i}(t), \\
& \text { where } 0<a<1 \\
& P_{j}(t+1)=\frac{a}{m-1}+(1-a) P_{j}(t), \\
& \text { where } i \neq j \text { and } j=1,2, \ldots, m
\end{aligned}
$$

It can be observed that the probability increases as the number of attempts increases and decreases with the increase in the number of accesses.

\subsection{Packets Queuing based on the Delay-Timer Value}

The sensor nodes in the network are considered to be mobile in nature. Hence the topology of the network is dynamic. The proposed algorithm considers various applications. The delay that the applications can tolerate varies from each other. A new field called Delay-Timer is added to the packet which is initialized with the delay value that the packet can tolerate. The Delay-Timer value is excluding the time taken by packet to be transmitted from the source to the destination. If the Delay-Timer is expired before it is transmitted, then it is considered as packet-drop. The goal of the proposed protocol is to reduce the number of packet drops and hence a queue is maintained where the packets are inserted according to the remaining Delay-Timer. For ex: if a packet with Delay-Timer $10 \mathrm{~ms}$ is in the queue and a packet with Delay-Timer $5 \mathrm{~ms}$ is arrived, then the later packet is serviced first. Suppose that the difference between the Delay-Timer of the two packets is less than $\alpha \mathrm{msec}$, the former is serviced first and then the later. The value of $\alpha$ is determined based on various runs while performing the experiment. The difference of $\alpha$ helps in eliminating the unnecessary drop of packets that are in the queue for the service. The procedure for queuing the packets is shown in the Figure.1. In the Figure.1, DNew represents the tolerable delay of the new packet. $D Q[i]$ represents the delay of the $i^{\text {th }}$ packet in the queue. $P Q[j]$ represents the packet in the $j^{\text {th }}$ index of the queue

\subsection{Selection of node based on the Success Rate}

Two different counters are maintained for each node in the network, $\mathrm{N}_{\text {access }}$ and $\mathrm{N}_{\text {attempt }}$. $\mathrm{N}_{\text {access }}$ is incremented when the node acquired a channel access and $\mathrm{N}_{\text {attempt }}$ is incremented when the node tried for a channel access irrespective of the success or failure. Another parameter called sequence number is maintained. The sequence number is the maximum number of times the node can access the channel.

Naccess - the number of times the node acquired the access to the channel

Nattempt - the number of times the node tried to acquire the channel access.

Hence, 
The success rate at which the node acquired the channel $=\frac{N_{\text {access }}}{N_{\text {attempt }}} \times 100$
The node whose Naccess < sequence number and the success rate is low will acquire the channel. The process is shown in Figure. 2

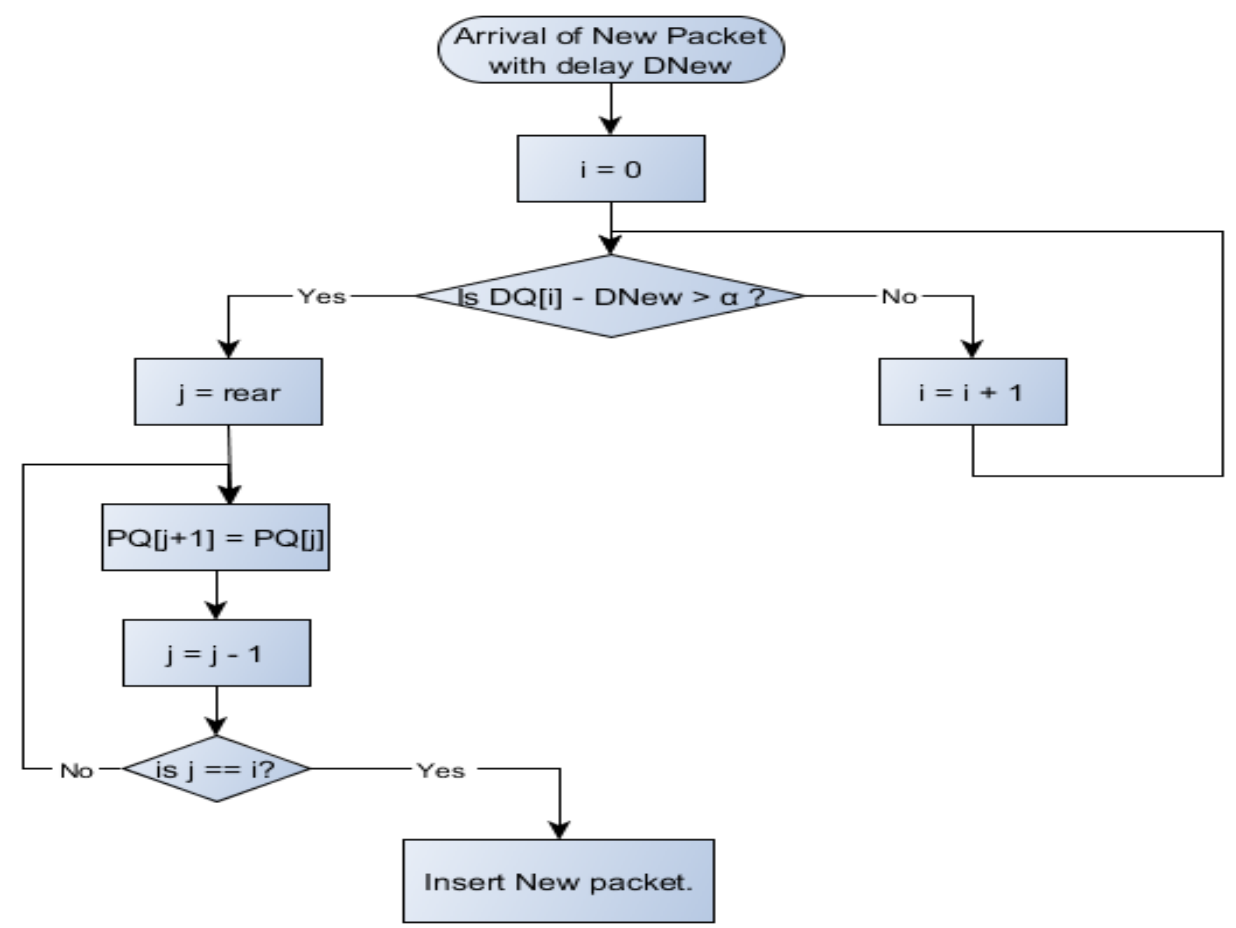

Figure.1 Flow diagram for packet queuing based on delay timer

\subsection{Selection of forwarding node based on the goodness value}

The ant colony optimization algorithm is used to enhance the performance of the algorithm. The node among many forwarding nodes is determined based on the goodness value of the node. The goodness value of the node is increased if the node is successfully forwarding the packet to the next node. The node with highest goodness value is chosen as a next forwarding node. As a particular node is chosen as forwarding node for more number of times then the pheromone value which is referred as goodness value increases automatically. The process of updating the goodness value of the node is shown in Figure. 3

Steps in identifying the next forwarding node: 1.Determine all the possible neighbours of a node \{Neighbours\}

2.Determine all the possible neighbours in the direction of the destination - \{Neigh-destdirection $\}$

a. \{Neigh-dest-direction $\}$ \{Neighbours $\}$

3.Obtain the goodness value of the nodes in $\{$ Neighdest-direction

4.The node with highest goodness value becomes the forwarding node. 


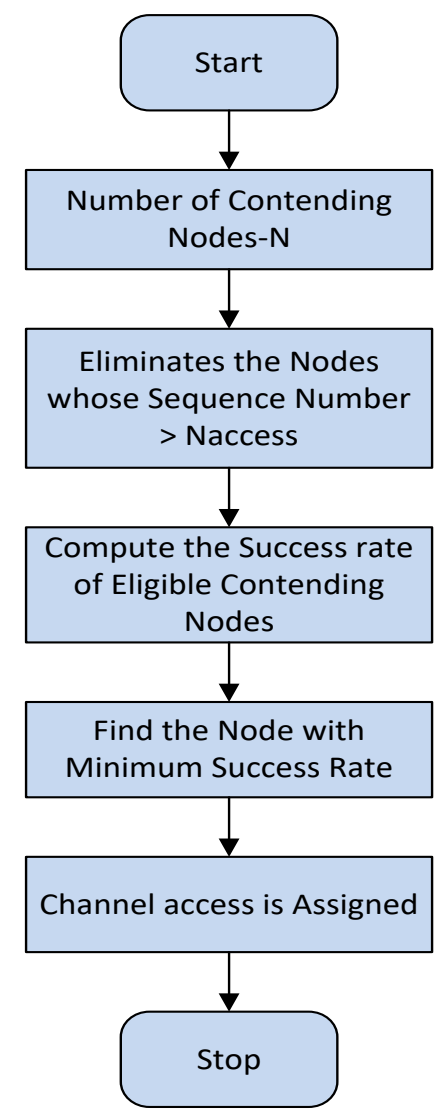

Figure. 2 Flow diagram of selecting a node based on the success rate

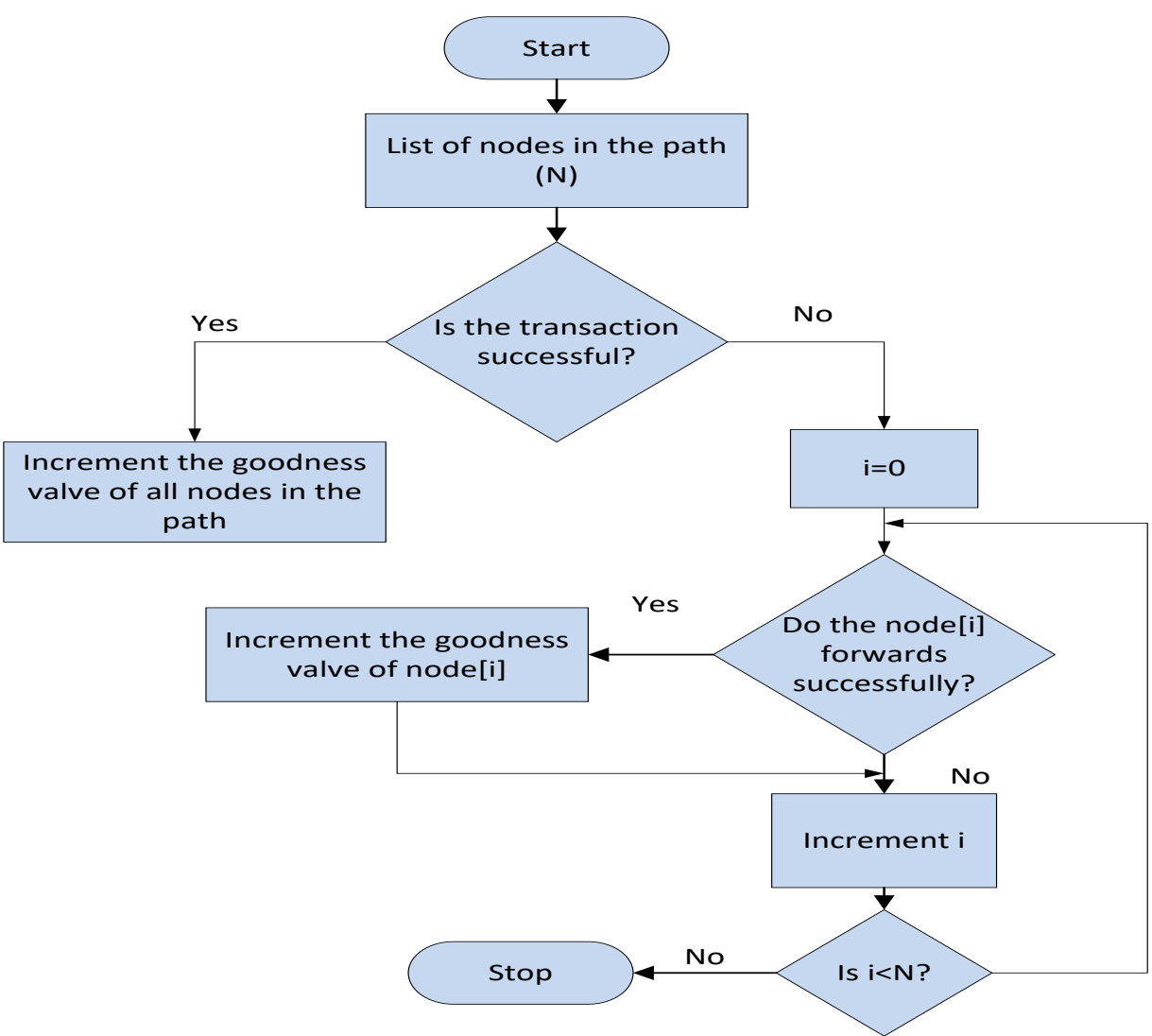

Figure.3 Flow diagram for updating the goodness value of the Node 


\subsection{Sleep Scheduling}

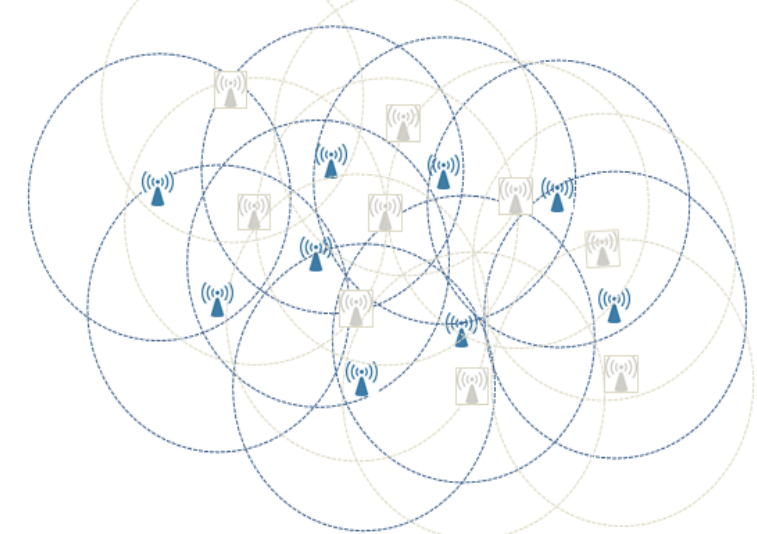

Figure.4 Wireless Sensor Network with group 1 in active state and group 2 in sleep mode

A node can go into the sleep mode for energy conservation. The nodes in the network are grouped into two such that the nodes in the group1 can communicate with all the remaining nodes in the group1 with/without the help of the nodes in the group2 and vice-versa. The nodes of group1 and group2 alternatively go to the sleep mode as shown in Fig. 4. When the nodes in the group1 are active, still some of the nodes remain idle with acting as a source, forwarding and destination nodes. In such a case, a node goes to the sleep state for $\Omega$ period of time after being idle for $\beta$ time. After $\Omega$ period of time, the radios are automatically turned on. When a particular group of nodes are in sleep mode, all nodes in that group are in sleep mode only and all the nodes awake from the sleep mode at the same time. The nodes can listen, transmit, forward or receive only when it is active. When a node wants to transmit some packets but if it do not get a chance of transmitting, then the packets are buffered and are transmitted when it is ready.

When more number of nodes are contending for the channel access, then a particular node is given the channel access according to the proposed algorithm as follows:

Initially the node is selected based on the delaysensitivity of the packets to be transmitted. If more such nodes are determined, then the success-rate of the node is considered and the node with less successrate is given a chance to acquire the channel access. If the node is a forwarding node, the selection depends both on the success-rate and the goodness value.

\section{Experimental Evaluation}

Table 1. Simulation Parameters

\begin{tabular}{|c|c|}
\hline Parameter & Value \\
\hline $\begin{array}{c}\text { Number of Contending } \\
\text { Stations }\end{array}$ & $2-200$ \\
\hline Sequence number & $50,100,150,200,250,300$ \\
\hline $\mathrm{CW} \min$ & 31 \\
\hline $\mathrm{CW} \max$ & 1023 \\
\hline $\mathrm{A}$ & $10 \mathrm{~ns}$ \\
\hline DIFS & $50 \mu \mathrm{s}$ \\
\hline SIFS & $10 \mu \mathrm{s}$ \\
\hline PHY data rate & $1 \mathrm{Mbps}$ \\
\hline PLCP Preamble & $144 \mu \mathrm{s}$ \\
\hline PLCP Header & $48 \mu \mathrm{s}$ \\
\hline Agent & UDP \\
\hline Packet size & 1200 \\
\hline Application & CBR \\
\hline CBR Rate & 64kbps \\
\hline Channel type & Wireless channel \\
\hline Radio-propagation model & Two ray ground \\
\hline Network interface type & WirelessPhy \\
\hline MAC Type & 802_11 DCF \\
\hline Interface Queue Type & Drop tail/priority queue \\
\hline Link layer type & LL \\
\hline Antenna Model & Omni antenna \\
\hline Routing protocol & AODV \\
\hline Topology & $650 * 750$ \\
\hline
\end{tabular}

The proposed algorithm, CLM-VBA is examined using the parameters - packet delivery ratio, end-toend delay, number of collisions and energy consumption. The enhancement of the packet delivery indirectly shows that the transmissions are successful and the minimization of collisions. The protocol is implemented and examined in ns-2 [26] and the results are taken from the average of 20 times of execution of the system. Parameters used in ns-2 simulation are shown in Table 1.

The DIFS and SIFS parameter values are used in order to setup the delay parameter. The performance of the ACTD-M-VBA is compared with the legacy systems like VBA, S-MAC and EACAODV. It can be observed from the graphs shown in Fig. 5 - Fig. 8 that the proposed algorithm ACTD-M-VBA outperforms when compared with the other algorithms. The VBA algorithm is developed for wireless networks. Sleep scheduling is employed and two counters are employed along with the sequence number for each node. There is a possibility of starvation of a node in the case of VBA. S-MAC is designed for MAC layer in wireless sensor networks. It introduced sleep scheduling as the energy need to 
be conserved in WSNs. EACAODV utilized ant colony optimization technique to enhance the performance of the system. The proposed algorithm employed queuing mechanism where packets are queued based on the delay that it can tolerate. This improves the quality of the packet delivery system. Success rate of each node is calculated based on the number of times it succeeded in acquiring the channel. This enhances the fairness allocation of a channel to a node among all the contending nodes and reduces collisions. The forwarding node is selected based on the goodness value defined for each node and which is altered based on the ant colony optimization technique. More pheromone implies high goodness value. This helps in increasing the packet delivery ratio and reducing the packet drops. Finally sleep scheduling is utilized in order to conserve the energy. As the alternate sets of nodes are being used, the lifetime of the network will be increased. The proposed algorithm reduces the delay by $44.44 \%$, $55.24 \%$ and $45.1 \%$ when compared to VBA, S-MAC and EACAODV respectively as shown in the Figure. 5. It can be observed from the Figure. 6, that $43.91 \%$, $56.71 \%$, and $20.88 \%$ enhancement is made in terms of packet delivery ratio by the ACTD-M-VBA in comparison with VBA, S-MAC and EACAODV respectively. The ACTD-M-VBA improves the performance by $32.9 \%, 29.06 \%$ and $9.06 \%$ in terms of average energy consumption when compared to the VBA, S-MAC and EACAODV respectively as shown in the Figure. 7. ACTD-M-VBA reduced the number of collisions by $28.2 \%, 45.67 \%$, and $35.98 \%$ in comparison to VBA, S-MAC and EACAODV respectively as it can be observed in Figure. 8.

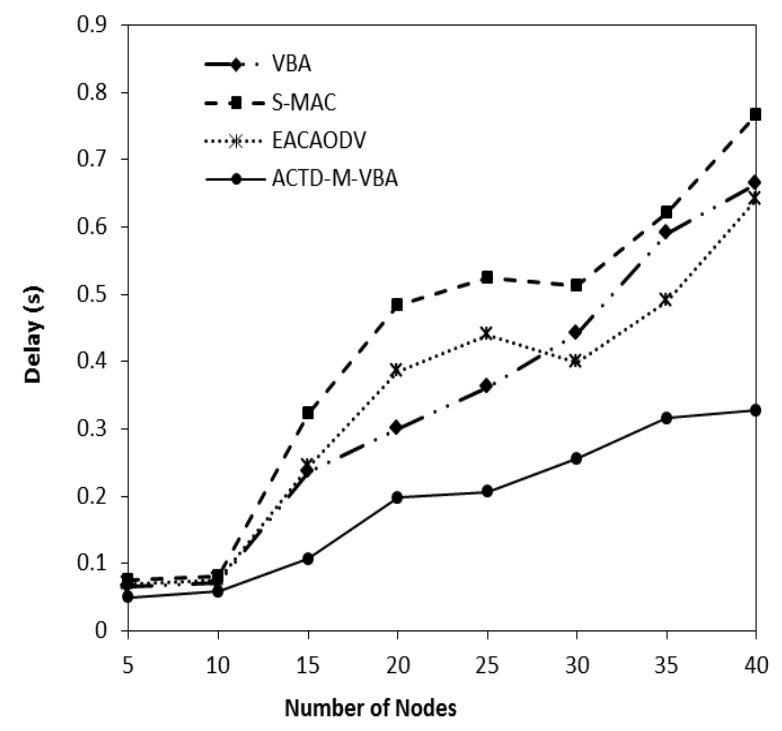

Figure.5 Delay vs Number of Nodes

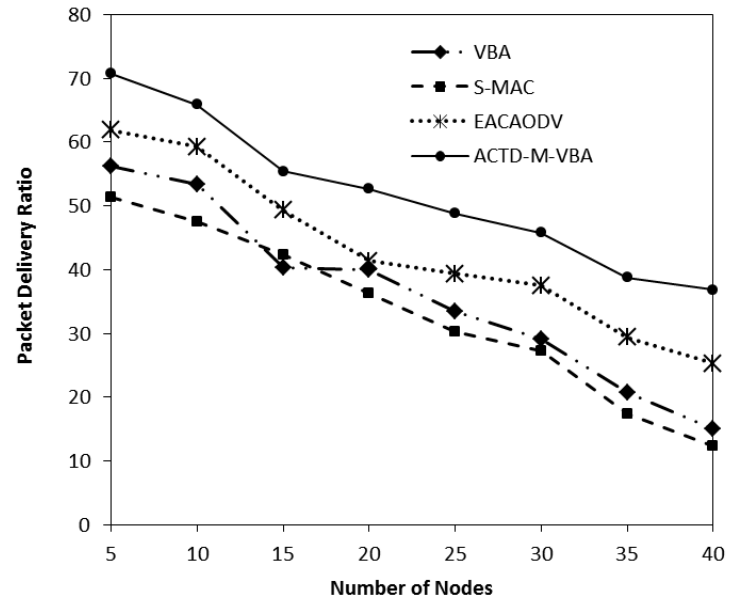

Figure.6 Packet Delivery Ratio vs Number of Nodes

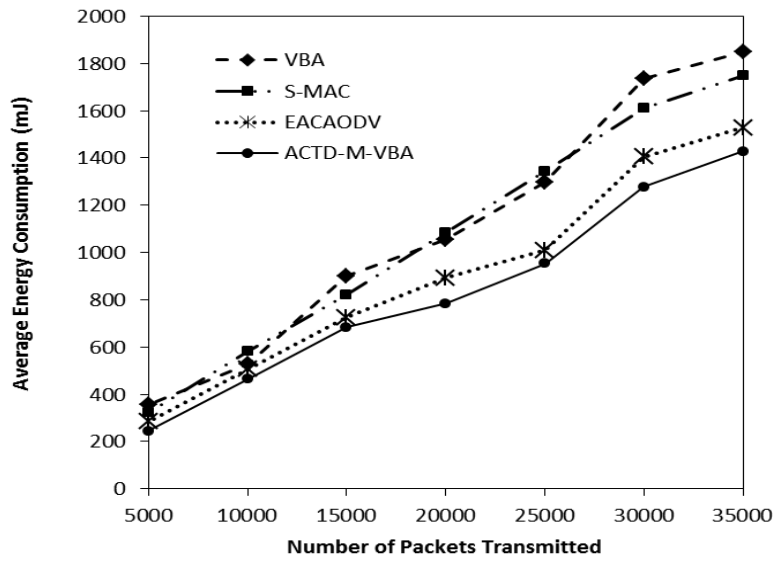

Figure.7 Average Energy Consumption vs Number of Packets Transmitted

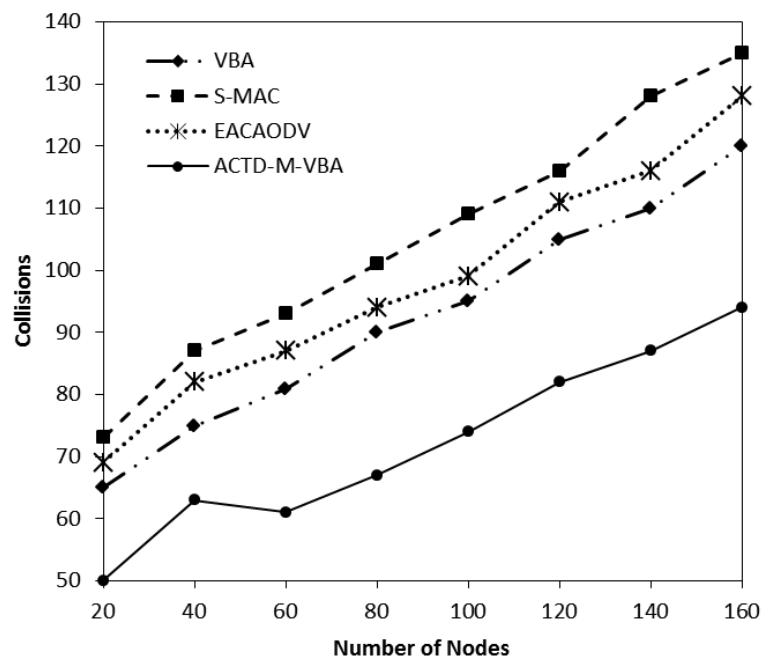

Figure.8 Number of Collisions vs Number of Nodes

\section{Conclusion}

In this paper, the performance of the network is enhanced in terms of packet delivery ratio, delay, average energy consumption and number of collisions. The priority is given to the delay sensitive 
applications and hence employed a queuing mechanism where the packets are queued based on the remaining tolerable delay and made the mechanism such that the packet is serviced before the delay that it can tolerate expires. The starvation problem and reduction of collisions are handled by computing the success rate of each node using the parameter maintained for keeping track of number of accesses and number of attempts that each node made to acquire the channel access. Energy consumption is reduced by sleep scheduling which describes that only a group of nodes in the network are used at a time. Then finally packet delivery ratio is increased by choosing the neighboring nodes among the available based on the goodness value which is being modified using the ant colony technique. The results shown prove that the proposed algorithm, ACTD-MVBA performs well when compared to VBA, SMAC and EACAODV. In the future, the proposed work is integrated with security parameters for enhancing the performance of the WSNs.

\section{References}

[1] Y. Wei, J. Heidemann and D. Estrin, "An EnergyEfficient MAC Protocol for Wireless Sensor Networks", IEEE INFOCOM, Vol. 2, pp. 1567-1576, 2002.

[2] G. J. Pottie and W. J. Kaiser, "Embedding the internet: wireless integrated network sensors", Communications of the ACM, Vol. 43, No. 5, pp. 5158, 2000.

[3] C. Intanagonwiwat, G. Ramesh, and E. Deborah, "Directed diffusion: A scalable and robust communication paradigm for sensor networks", in Proceedings of the ACM/IEEE International Conference on Mobile Computing and Networking, pp. 56-67, 2000.

[4] H. John, S. Fabio, C. Intanagonwiwat, G. Ramesh, E.Deborah, and G. Deepak, "Building efficient wireless sensor networks with low-level naming", in Proceedings of the Symposium on Operating Systems Principles, Lake Louise, Banff, Canada, Vol. 35, No. 5, pp. 146-159, 2001.

[5] Frazer Bennett, David Clarke, Joseph B. Evans, Andy Hopper, Alan Jones, and David Leask, "Piconet: Embedded mobile networking", IEEE Personal Communications Magazine, Vol. 4, No. 5, pp. 8-15, 1997.

[6] W. Alec and C. David, "A transmission control scheme for media access in sensor networks", in Proceedings of the ACM/IEEE International Conference on Mobile Computing and Networking, pp. 221-235, 2001.
[7] S. Parvatkar and D. Gore, "Energy Efficient Protocol for Heterogeneous Wireless Sensor Network using Ant Colony Optimization", International Journal of Computer Science and Information Technologies, Vol. 5,No. 3, pp. 3454-3456, 2014.

[8] S. Sendra, "Power Saving and Energy Optimization Techniques for Wireless Sensor Networks", Journal of communications, Vol. 6, No. 6, pp.439-459, 2011.

[9] M. N. Rahman, "Efficient Algorithm for Prolonging Network Lifetime of Wireless Sensor Network", Tsinghua Science and Technology, Vol. 16, No. 6, pp.561-568, 2011.

[10] L. Zhenjun, Z. Yingjiang and P. Zhangfan, "Research on Energy-Efficient Intelligent Method for WSN", Journal of Convergence Information Technology (JCIT), Vol. 8, No. 1, pp. 28-36, 2013.

[11]F. Shraddha and K. Deepak, "Energy Efficient Resource Allocation in Wireless Sensor Networks", International Journal of Computer Science and Mobile Computing, Vol.3, No.5, pp. 887-892, 2014.

[12] W. Ye, J. Heidemann, and D. Estrin,, "Medium access control with coordinated adaptive sleeping for wireless sensor networks", IEEE/ACM Transactions on Networking, pp. 493-506, 2004.

[13] T. V. Dam, and K. Langendoen, “An adaptive energy efficient MAC protocol for wireless sensor networks", In Proceedings of the 1st international conference on Embedded networked sensor systems, pp. 171-180, 2003.

[14] P. Lin, C. Qiao, and X. Wang, "Medium access control with a dynamic duty cycle for sensor networks", IEEE Wireless Communications and Networking Conference, pp. 1534-1539, 2004.

[15]C. Suh, and Y.B. Ko, "A traffic aware, energy efficient MAC protocol for wireless sensor networks", In Proc. of the IEEE international symposium on circuits and systems (ISCAS'05) Vol. 3, pp. 29752978, 2005.

[16] S. Pranesh and P. Jae-Young, "Medium reservation based sensor MAC protocol for low latency and high energy efficiency", Telecommunication Systems, Vol. 52, pp. 2387-2395, 2013.

[17] A. Attiah, T.A. Mustafa, M. Chatterjee and D. Turgut "EE-MAC: Energy efficient sensor MAC layer protocol", 9th IEEE International Workshop on Performance and Management of Wireless and Mobile Networks, pp. 116-119, 2013.

[18] P. Krishna, S. Misra, M. S. Obaidat, and V. Saritha, "Virtual backoff algorithm: an enhancement to 802.11 medium-access control to improve the performance of wireless networks," IEEE Trans. Veh. Technol., Vol. 59, No. 3, pp. 1068-1075, 2010.

[19] N. Aydin, S. H. You, B. Turgut, and D. Turgut, "Scenario-based performance analysis of MAC protocols for wireless sensor networks," in 
International Conference on Wireless Networks (ICWN), pp. 105-111, 2005.

[20] S. De, C. Qiao, D. Pados, M. Chatterjee, and S. Philip, "An integrated cross-layer study of wireless CDMA sensor networks," IEEE Journal on Selected Areas on Communications (JSAC), Special Issue on Quality of Service Delivery in Variable Topology Networks, Vol. 22, pp. 193-205, 2004.

[21] S. Kumar, "A Survey on Scheduling Algorithms for Wireless Sensor Networks", Journal of Communications, Vol. 20, No. 5, pp. 12-32, 2011.

[22] L. Freeny, "Energy Efficient Communication in Ad hoc Networks", Mobile Ad hoc Networking, pp. 301328, 2004.

[23]N. K. Ray, "A Review on Energy Efficient MAC Protocols for Wireless LANs", in 2009 International Conference on Industrial and Information Systems (ICIIS), pp. 137-142, 2009.

[24]D. Kim, J. Kim and K. Park, “An event-aware MAC scheduling for energy efficient aggregation in wireless sensor networks", Computer Networks, Vol. 55, pp.225-240, 2011.

[25] S. Dayang, Y. dong and W. Aimin, "Lifetime Optimizing Scheme of WSN", Journal of Computer Research and Development, Vol. 1, pp. 193-201, 2012.

[26] W. Chen and L. Cheng, "Cross-layer energy efficient MAC protocol for wireless sensor networks", Computer Engineering and Applications, Vol. 27, No. 10, pp. 102 - 106, 2011.

[27] J. Zhong and Z. Jun, "Energy-efficient local wake-up scheduling in wireless sensor networks", Evolutionary Computation (CEC), 2011 IEEE Congress on , pp. 2280-2284, 2011

[28] B. Jiang, B. Ravindran, and H. Cho, "Energy Efficient Sleep Scheduling in Sensor Networks for Multiple Target Tracking', Distributed Computing in Sensor Systems, Lecture Notes in Computer Science, Vol. 50, No. 67, pp. 498-509, 2008.

[29]C. Suh, and Y.B. Ko, "A traffic aware, energy efficient MAC protocol for wireless sensor networks", In Proc. of the IEEE international symposium on circuits and systems (ISCAS'05), Vol. 3, pp. 29752978, 2005. 